Olivier SIMONIN

CRESEM, Université de Perpignan - Via Domitia

\title{
L'aventure de Hautdesert et les chasses de Bertilak
}

Sir Gawain and the Green Knight ${ }^{1}$ entrelace de nombreux fils, à la fois narratifs et thématiques, qui se nouent et se recouvrent avec art. A cet égard, l'aventure de Hautdesert - comme nous conviendrons de nommer le séjour en partie périlleux de Gauvain dans ce château - et son épilogue à la chapelle verte présentent un intérêt particulier, et les scènes de chasse du seigneur Bertilak livrent de précieuses clefs pour bien saisir la portée de cet épisode.

Afin d'analyser les scènes de chasse et les épisodes qui lui sont liés, donnant lieu à des lectures convergentes, à une confluence interprétative, nous allons contextualiser l'aventure de Gauvain au château de Bertilak, passer en revue les écrits critiques sur ses chasses, puis établir à partir d'elles tout un dense réseau de résonnances signifiantes.

\section{CONTEXTUALISATION : L'AVENTURE DE HAUTDESERT}

L'aventure de Hautdesert se voit accordée une place privilégiée dans le roman. Les hôtes de Gauvain entreprennent de le piéger, et de nombreux indices textuels soulignent son insuffisance et anticipent son échec.

\section{Le piège de Hautdesert}

Les trois scènes de chasse de Bertilak encadrent littéralement les scènes de tentation de Gauvain, où la châtelaine, l'épouse de son hôte, met la vertu du

\footnotetext{
${ }^{1}$ Les références au texte suivent l'édition de Malcom Andrew et Ronald A. Waldron, The Poems of the Pearl Manuscript, 4 édition, Exeter: University of Exeter Press, [1987] 2002.
} 
chevalier à rude épreuve en entreprenant de le séduire. Ces scènes doubles, présentées sur le mode du contrepoint, se voient clôturées chaque jour par une scène de cour où Bertilak et Gauvain se retrouvent en outre pour échanger leurs gains du jour, suivant un vœu pris conjointement. Cet ensemble de scènes constitue la troisième des quatre grandes parties (fitts) du poème reconnues dans les éditions modernes. Il s'agit de la partie la plus longue (872 vers sur un total de 2531), qui revêt de fait une importance considérable.

C'est ainsi logiquement au château de Bertilak, à Hautdesert, que se déroule l'aventure principale du roman en vers : les trois tentations de Gauvain sont de véritables tests dont découlent l'issue de sa rencontre finale avec le chevalier vert (qui n'est autre que Bertilak), qui s'essayera trois fois à décapiter Gauvain, ne lui coupant qu'un peu de peau au dernier coup. La coupure reflète l'échec partiel du neveu d'Arthur, qui a finalement accepté de la châtelaine une ceinture verte censée le protéger des coups dans l'objectif de préserver sa vie lors de son rendez-vous funeste avec le chevalier vert.

Comment Gauvain est-il mis à l'épreuve ? Outre les avances de la châtelaine, que le chevalier se doit de rejeter avec courtoisie, il se trouve contraint de participer à un échange de gains, proposé par son hôte comme un jeu : ce que, pendant trois jours, Bertilak obtiendra de ses chasses, il fait vœu de le donner à son invité, qui devra faire de même pour ce qu'il aura obtenu au château, où le seigneur l'enjoint de demeurer afin de se reposer. Il rendra au seigneur les baisers prodigués par la châtelaine, mais faillira en ne restituant pas la ceinture verte.

Les tentatives pour piéger Gauvain sont orchestrées par Morgane, comme l'apprend le lecteur ou l'auditeur en fin de roman, mais elles sont aussi mises en œuvre par Bertilak et sa femme, au cours de l'aventure de Hautdesert. Une des stratégies à laquelle recourent ces derniers consiste à endormir la vigilance de Gauvain pour qu'il baisse sa garde dans les tests de vertu cruciaux auxquels il est soumis, sans en être préalablement avisé. La critique a ainsi pu souligner 
l'usage du vin visant à émousser l'attention du chevalier, à le rendre plus vulnérable. ${ }^{2}$ Les références au vin, au fait de boire et à la boisson sont au nombre de dix durant l'épisode de Hautdesert (v. 900, 980, 1025, 1112, 1403, $1409,1668,1684,1750,1935)$. Il n'est donc pas surprenant que dès l'ouverture de la première scène courtoise où la châtelaine tente de séduire Gauvain, celui-ci se trouve dans un état de somnolence (v. 1178-1183) :

Pus laykes pis lorde by lynde-wodez evez

And Gawayn pe god mon in gay bed lygez,

Lurrkez quyl pe daylyzt lemed on pe wowes,

Under couertuour ful clere, cortyned aboute.

And as in slomeryng he slode slezly he herde

A little dyn at his dor and derfly vpon; ${ }^{3}$

Ce qui frappe naturellement ici, c'est que le chevalier d'Arthur est présenté comme inactif, apparaissant dans un rôle inhabituel, à contre-emploi. Son inactivité est rendue d'autant plus saillante que les scènes où il figure se trouvent juxtaposées avec les scènes de chasse, suggérant au passage une comparaison implicite entre les activités cynégétiques de Bertilak et le repos tout relatif de Gauvain dans son lit. Dans le château où il subit sans le vouloir une série d'épreuves, d'une façon qui n'est pas sans évoquer les aventures des chevaliers d'Arthur au château du Graal, ses hôtes cherchent à le surprendre afin de le prendre à défaut.

\footnotetext{
${ }^{2}$ Ad Putter, An Introduction to the Gawain-Poet. Londres and New York: Longman, 1996, p. 84.

Ainsi donc s'amusait le seigneur à la lisière du bois,

Et Gauvain, le brave homme, était couché dans un beau lit,

Blotti jusqu'à ce que la lumière du jour luise sur les murs,

Sous la couverture tout éclatante, tout entouré de courtines ;

Dans un demi-sommeil il entendit doucement

Un petit bruit à la porte, qui s'ouvrit furtivement ;

Comme pour les autres citations de l'œuvre, la traduction en français que nous avons choisie (avec des annotations entre crochets pour préciser le sens littéral) est la suivante : Juliette Dor, Sire Gauvain et le chevalier vert, Paris : 10/18, "bibliothèque médiévale", 1993.
} 
Les perturbations de Gauvain ne sont en fait pas seulement liées à l'alcool, comme le démontre le début de la troisième scène courtoise (v. 17461752):

'A ! mon, how may pou slepe?

Pis morning is so clere.'

He watz in drowping depe,

Bot penne he con hir here.

In dre3 droupyng of dreme draueled pat noble,

As mon pat watz in morning of mony pro poztes,

How pat Destiné schulde pat day dele hym his wyrde

At pe Grene Chapel when he Pe gome metes ${ }^{4}$

Les sombres pensées qui tournent dans l'esprit de Gauvain constituent une diversion supplémentaire susceptible d'endormir son attention pour une épreuve qui, bien qu'elle paraisse secondaire, détermine en fin de compte le succès de sa rencontre à venir avec le chevalier vert, qui le hante. C'est là un paradoxe. De manière tout à fait semblable, dans la deuxième scène courtoise, la châtelaine lui rend visite pour alléger sa sombre humeur, His mode for to remwe (v. 1475), ce qui est traditionnellement le rôle du vin, des fêtes et des jeux, qui sont à l'honneur au château de Bertilak. Tous ces éléments participent d'une stratégie de distraction et de dévoiement de Gauvain par détournement de sa vigilance en vue de le prendre au piège, comme du vulgaire gibier.

\footnotetext{
$4 \quad$ "Ah quel homme ! Comment peux-tu dormir

Par ce clair matin ?"

Il était dans une profonde torpeur,

Mais pourtant il l'entendit.
}

Dans la lourde torpeur de son rêve, le noble chevalier murmurait,

Comme un homme perturbé par des pensées oppressantes :

Comme la Destinée allait-elle régler son sort en ce jour

A la chapelle verte, lorsqu'il rencontrerait le chevalier, 


\section{Prolepses et ironies}

De nombreux éléments proleptiques empreints d'une ironie narrative certaine annoncent la chute du protagoniste. La symbolique des nombres, prégnante dans tout le poème, oppose le chiffre trois à cinq, celui de la perfection voulue par le neveu d'Arthur et figurée sur son bouclier sous la forme du pentacle. ${ }^{5}$ Le chiffre trois est lié à plusieurs reprises à la perte de Gauvain, ne serait-ce qu'au jeu qu'on lui impose à Hautdesert, qui engage sa parole. Ainsi, en prélude et à la veille du troisième jour, qui est le jour où le chevalier faillit, son hôte l'encourage (v. 1680) : Now "Prid tyme, prowe best" penk on pe morne. ${ }^{6}$

En rappelant un proverbe de jeu, en (moyen) anglais, "La troisième, c'est la bonne", Bertilak laisse entendre qu'il espère prendre au piège son invité comme à l'occasion d'un intermède ludique, tout en disant explicitement une tout autre chose. La trahison de Pierre qui renie le Christ est évoquée par les trois chants du coq qui n'arrivent pas, en tant que série, jusqu'à terme, avant le départ de Bertilak pour la deuxième chasse (v. 1412): Bi pat pe coke had crowen and cakled bot pryse. ${ }^{7}$ Cela signifie certainement que Gauvain n'est pas encore mûr pour tomber. En revanche, il l'est juste avant son départ pour la chapelle verte, puisqu'il entend avant son lever des coqs chanter (v. 2008), soit trois fois au cours de la nuit suivant la croyance populaire médiévale. ${ }^{8}$

Le chevalier vient alors de rendre à Bertilak les trois baisers reçus de la châtelaine, ayant omis de lui remettre la ceinture verte et même de la mentionner. Ces baisers rappellent naturellement celui Judas, leur nombre faisant écho au reniement de Pierre. Qui plus est, de nombreux signes renvoient aux enjeux de la venue du neveu d'Arthur à la chapelle verte et à sa très

\footnotetext{
${ }^{5}$ Olivier Simonin, "La figure de la Vierge dans Sire Gauvain et le chevalier vert", dans La Vierge dans les arts et les littératures du moyen âge, éd. P. Bretel, Paris : Champion, à paraître.

${ }^{6}$ Rappelle-toi demain que "c'est la troisième fois qui est la bonne",

${ }^{7}$ Avant que le coq n'eût chanté et coqueriqué trois fois,

${ }^{8}$ Malcom Andrew and Ronald A. Waldron, op. cit., p. 280.
} 
probable issue : le motif de la décapitation est omniprésent. Il est déjà entrevu lors d'un des défis lancé par Bertilak dans l'esprit d'un amusement durant la veille de Noël (v. 983-985) :

\section{Hent hezly of his hode and on spere henged}

And wayned hom to wynne pe worchip perof

Pat most myrbe myzt meue pat Crystenmas whyle. ${ }^{9}$

Le capuchon est associé au chef par métonymie. ${ }^{10}$ Il est présenté sur le bout d'une pique ou d'un épieu, comme s'il s'agissait d'une tête prise comme trophée. L'espace non rempli sous le capuchon suggère en creux celle de Gauvain, en attente d'une décollation plus que probable. Le motif réapparaît de façon insistante lors du dépeçage de la première journée avec le découpage des têtes de cervidés (v. 1330 ; 1353-1355), et surtout avec celle du sanglier, que Bertilak offre en cadeau à Gauvain (v. 1593, 1607, 1616, 1633, 1635). Ce don peut également se lire comme une provocation à un niveau symbolique, alors inaccessible au chevalier.

À l'encontre de Gauvain et de ce qu'il représente, la moquerie parait être érigée en loi par les maîtres de Hautdesert. Le rapport de vassalité est parodié à plusieurs reprises. Lorsque Gauvain donne des baisers à son hôte en échange des prises de chasse, il se met dans une situation délicate, et ce dès le premier échange (v. 1388-1389) :

He hasppez his fayre hals his armez wythinne And kisses hym as comlyly as he coupe awyse: ${ }^{11}$

\footnotetext{
${ }^{9}$ Saisit gaiement son capuchon, l'accroche à une lance, Et les défie de se l'approprier :

C'est à celui qui déploiera le plus de rires en cette nuit de la Nativité.

${ }^{10}$ Claire Vial, "Fêtes et saisons dans Sir Gawain and the Green Knight", dans Sir Gawain and the Green Knight. Essays and Studies, André Crépin et Colette Stévanovitch (éds), Paris, Publications de l'AMAES, pp. 61-83, 1994; Blaise DOUGLAS, "Transparence narrative et apparences faussement trompeuses dans Sir Gawain and the Green Knight", dans Transparences médiévales, Martine Yvernault (éd.), Limoges : PULIM, 2015, p. 69.

${ }^{11}$ Il entoure le beau cou de son hôte dans ses bras,
} 
$\mathrm{Au}$ moyen âge, le baiser pouvait être un geste public, notamment pour faire acte d'hommage. À travers ces deux vers transparaît la gêne de Gauvain, qui doit rendre un geste d'ordre privé en public, dont la réalisation dans ce contexte équivaut à une soumission qui n'est nullement appropriée. Il n'est en passant pas anodin que la partie du corps qu'est le cou soit explicitement mentionnée ici. En plus d'une nouvelle allusion au sort de Gauvain et à l'endroit où se pratique la décollation, le coup reçu de Bertilak à la chapelle verte évoque la collée d'un adoubement (au cours duquel l'impétrant donne également un baiser).

Concernant l'idéal courtois qu'incarne le neveu d'Arthur, une vision au rabais ou simplement biaisée en est proposée par la châtelaine, qui prend l'initiative dans le jeu amoureux, tout comme Bertilak prend l'initiative en faisant diverses propositions à Gauvain qu'il ne peut pas refuser à son hôte, comme rester au lit tandis que lui chasse. Cela contredit de façon évidente les règles courtoises, qui se voient subverties voire inversées. ${ }^{12}$

De manière plus générale, les sous-entendus de nature sexuelle qui s'accumulent au fil du texte ternissent l'image noble du parangon courtois et pointent vers des significations peu flatteuses, suggérant un chevalier féminisé, passif, voire paresseux et luxurieux. En surplus des conversations avec la châtelaine, dont la direction des propos ambigus ne laisse en réalité aucun doute quant à leur teneur charnelle, la ceinture, le nœud que Gauvain reçoit appartient au domaine féminin et dénote par métonymie et similitude l'organe sexuel lui étant propre. Le fait que Gauvain le porte lors de son rendez-vous avec le chevalier vert est pour le moins significatif. Il y subit une circoncision symbolique le Jour de l'An (jour fête de la Circoncision) en recevant le coup de

\footnotetext{
Et l'embrasse avec toute la bienséance qu'il peut imaginer.

${ }^{12}$ Voir à ce sujet : Olivier Simonin, "Engagements de Gauvain et courtoisie dans Sir Gawain and the Green Knight", dans Publication de L'Association des Médiévistes Anglicistes de L’Enseignement Supérieur 37, Nolwena Monnier (éd.), pp. 123-144, Paris, 2016.
} 
hache qui lui coupe un morceau de peau au niveau du cou. ${ }^{13}$ Juste après cette ablation emblématique, il se lance dans le lieu médiéval très commun du blâme des femmes auprès de Bertilak, évoquant toute une série de personnages masculins de l'Ancien Testament, trahis par des femmes avec lesquels ils se sont adonnés au plaisir charnel, pourtant évité par le chevalier. ${ }^{14}$

Dans le poème, Gauvain est un chevalier religieux, chrétien, protégé par la Vierge. Or les fêtes et les festins de cour, et de manière privilégiée à Hautdesert, s'approchent potentiellement de parodies de l'Eucharistie, où le vin et la nourriture sont en surabondance. Le détournement du religieux est particulièrement flagrant lors de l'arrivée de Gauvain, où il se voit offrir (le vendredi ?) une débauche de plats de poisson et leurs accompagnements, tandis que Bertilak s'excuse de la maigreur de la chère (v. 897-898), qu'il désigne sous le terme de pénitence :

'Pis penaunce now ze take

And eft hit schal amende. ${ }^{, 15}$

Cela soulève la question de la relation entre les éléments féériques, voire païens, d'une part, et chrétiens d'autre part, à Hautdesert. Nous laisserons celleci de côté pour revenir aux affronts symboliques qu'y subit Gauvain, entravé par les règles de la parole et réduit à l'inaction. L'opprobre qu'il connaît est, en un sens, semblable à celle de Lancelot dans Le Chevalier à la charrette, mais, contrairement à lui, son humiliation est en grande partie proleptique, construite par l'intermédiaire d'un réseau de signes et de références. Elle contribue à remettre en question sur le mode ironique voire parodique la compatibilité des

\footnotetext{
${ }^{13}$ Robert J. Blanch, "Religion and Law in Sir Gawain and the Green Knight", Approaches to Teaching Sir Gawain and the Green Knight, éd. M. Youngerman Miller et J. Chance, New York: The Modern Language Association of America, 1986, p. 94.

${ }^{14}$ La moquerie se situe alors à un niveau textuel, dépassant les manigances de Bertilak.

15 "Acceptez maintenant cette pénitence,

Cela s'améliorera plus tard."
} 
idéaux concentrés en la figure de Gauvain, plutôt que de jauger sa résolution à servir sa dame ou sa reine.

\section{PISTES CRITIQUES: LES TROIS CHASSES DE BERTILAK}

L'enchâssement des scènes courtoises à l'intérieur de la narration des chasses de Bertilak est un procédé qui attire l'attention. La question de leur signification et du commentaire éventuel qu'elles pourraient apporter aux premières a été soulevée à de nombreuses reprises, et nous aborderons ici les études précédentes à ce sujet d'un point de vue chronologique, tout en nous laissant la liberté de suivre certaines des pistes ouvertes en proposant une réflexion complémentaire.

\section{Une analyse métaphorique des animaux chassés}

Selon Henry Lyttleton Savage, ${ }^{16}$ le premier à notre connaissance à se prononcer sur le sujet avec quelque précision et prolixité, les animaux chassés représentent la manière dont Gauvain réagit aux avances de la châtelaine : de manière précautionneuse tout d'abord comme les cervidés, de manière brutale voire impétueuse comme le sanglier ensuite et de manière rusée comme le renard pour finir. Pour illustrer le propos, le deuxième jour où Bertilak chasse le sanglier, le dialogue entre sa femme et Gauvain tournerait ainsi autour des notions de force et de violence, comme lorsque la fougueuse dame tance le chevalier (v. 1495-1497) - qui répondra d'ailleurs de façon jugée abrupte selon Savage :

'Ma fay,' quop pe meré wyf, 'ze may not be werned;

Zif ar stif innoghe to constrayne wyth strenkpe, zif yow likez, Zif any were so vilanous pat uow devaye wolde. ${ }^{17}$

\footnotetext{
${ }^{16}$ Henry Lyttleton Savage, The Gawain-Poet: Studies in his Personality and Background, Chapel Hill: The University of North Carolina Press, 1956.

17 "Ma foi", dit la joyeuse femme, "on ne peut vous refuser,

Vous êtes suffisamment fort pour contraindre par la force si vous le voulez,
} 
L'idée est intéressante, mais force est de constater que la notion de violence ou de puissance s'applique au moins tout autant à la châtelaine. De plus, d'autres critiques ont vu différents péchés ou traits de Gauvain symbolisés par les animaux chassés : la témérité ${ }^{18}$ ou l'orgueil mêlé d'un appétit excessif pour la chair (luxure et gourmandise), ${ }^{19}$ par exemple, pour reprendre le cas du sanglier. La diversité des interprétations invite donc à la circonspection, comme le notent certains. ${ }^{20}$

L'autre point essentiel abordé par Savage (et repris par d'autres, comme Débax), est que la noblesse décroissante des animaux chassés entretient un rapport inversement proportionnel aux gains croissants du neveu d'Arthur. En effet, suivant les manuels cynégétiques médiévaux, les bêtes les plus nobles sont les bêtes de vénerie que sont les cervidés, alors que les bêtes de chasse auxquelles appartiennent le sanglier et le renard correspondent à une classe inférieure, au sein de laquelle le renard est jugé inférieur au sanglier. Comment interpréter cela? Il nous apparaît que la décroissance des gains de Gauvain mime sa chute, tandis que la croissance des baisers obtenus suggère, en parallèle, un gain en vice, en passivité voire en féminité.

\section{La métaphore de la chasse}

Dans son étude sur la chasse dans la littérature médiévale, ${ }^{21}$ Marcelle Thiébaux s'intéresse en premier lieu à la chasse prise comme métaphore de

\footnotetext{
A supposer que quelqu'un ait la vilenie de vous repousser."

18 Muriel Ingham and Lawrence Barkley, "Further animal parallels in Sir Gawain and the Green Knight", dans Chaucer Review 13, pp. 284-386, 1979.

${ }^{19}$ Joseph A. Longo, "Sir Gawain and the Green Knight: The Christian Quest for Perfection", in Nottingham Medieval Studies 11, pp. 57-85, 1967.

${ }^{20}$ Anne Rooney, "The Hunts in Sir Gawain and the Green Knight", dans A Companion to the Gawain-Poet, Derek S. Brewer \& Jonathan Gibson (éds), Woodbridge, Rochester (New York): D. S. Brewer, 1997, pp. 157-163.

${ }^{21}$ Marcelle Thiébaux, The Stag of Love: The Chase in Medieval Literature, Ithaca (NY): Cornell University Press, 1974.
} 
l'amour, héritage d'Ovide, dont on connaît l'influence fondatrice sur le développement du modèle courtois. Deux citations de l'Ars Amatoria suffiront à illustrer l'emploi du trope par le poète latin :

La femme qui sera à ton goût, il faut la chercher.

Les filets pour les cerfs, le chasseur sait bien où les tendre ;

Dans quel vallon se trouve le sanglier qui grogne, il le sait |bien.

Les oiseleurs connaissent les taillis, le pêcheur à la ligne

Connaît les eaux où circulent de nombreux poissons ;

Toi aussi, qui cherches matière à un amour durable,

Apprends en quels lieux les filles sont légion.

(v. 44-50) $)^{22}$

Mais toi, tu chasseras surtout dans les méandres des théâtres :

Ces lieux sont plus giboyeux que tu ne peux le rêver.

(v. 89-90) $)^{23}$

L'auteur relève en particulier, dans le roman, l'emploi du vocabulaire de la chasse, qui est abondamment utilisé dans les scènes de séduction, afin de suggérer que Gauvain fait figure de proie. Ainsi, la châtelaine encourage le chevalier comme les meneurs de meute le font pour les chiens (rehayted: v. 1422, 1744) - celui-ci d'ailleurs, invité à se reposer en son lit, y demeure et

${ }^{22}$ Danièle Robert, Ovide : Ecrits érotiques, Paris : Actes Sud. 2003, p. 181. L'original figure p. 180 :

Qucerenda est oculis apta puella tuis.

Scit bene venator, cervis ubi retia tendat;

Scit bene, qua frendens valle moretur aper.

Aucupibus noti frutices, qui sustinet hamos

Novit quae multo pisce natentur aqua ;

Tu quoque, materiam longo qui quaris amori,

Ante frequens quo sit disce puella loco.

${ }^{23}$ Ibid., p. 183. L'original figure p. 182 :

Sed tu pracipue curvis venare theatris :

Hac loca sunt voto fertiliora tuo. 
même s'y tapit avant d'être dérangé par la femme de Bertilak, comme les cervidés de la première le sont au début de la première chasse, forcés à sortir de leurs cachettes. ${ }^{24}$ L'isotopie de la capture est également fortement représentée dans les scènes courtoises, notamment sous la forme de verbes (cache, fonge, take). ${ }^{25}$ L'analogie entre la chasse et la poursuite amoureuse - voire selon nous, les prises de chasse et les tentatives de prise au piège du chevalier - est patente ici, même si sa présence reste globalement discrète dans la poésie ricardienne.

\section{La figure du renard (et du veneur)}

L'analyse de Marcelle Thiébaux se poursuit avec l'étude de la figure du renard, et rejoint en cela le travail de Débax. ${ }^{26}$ Pour ce dernier, Gauvain serait in fine figuré par le renard de la dernière chasse, animal avec lequel il partage la ruse (en refusant les avances de la châtelaine tout en acceptant la ceinture, qu'il dissimule au seigneur Bertilak), alors qu'il ne possède aucunement les traits communément attribués au cerf et au sanglier, ce qui expliquerait qu'il ne commette pas de faute dans les deux premières scènes de tentation. La correspondance qui vaut finalement pour Gauvain se révèle être la moins glorieuse, puisque le renard est le plus vil animal chassé. À ce titre, il attire l'attention sur la fin peu glorieuse du renard :

Il [Renart] fuit devant la chasse ; il use de feintes pour échapper à ses poursuivants... Mais à la fin de la poursuite, Renart, qui se trouve en face du seigneur Bertilak et à portée de sa lame, fait un dernier écart pour échapper à ce que l'on pourrait appeler cette mort "noble", et cet écart même le conduit à sa perte, car il se projette dans la gueule du chien le plus proche... la mort de Renart, à la différence de celle des autres

\footnotetext{
${ }^{24}$ Marcelle Thiébaux, op. cit., pp. 74-75.

${ }^{25}$ Ibid., pp. 85-77.

${ }^{26}$ Jean-Paul Debax, "Chasse au goupil et valeurs courtoises dans Sire Gauvain et le chevalier vert", dans Sir Gawain and the Green Knight: Essays and Studies, André Crépin \& Colette Stévanovitch (éds), Paris : publications de l'AMAES, 1994.
} 
animaux, n'est pas rapportée, car elle n'en vaut pas la peine ; ni non plus son dépeçage, qui n'a pas lieu, mais qui est remplacé par un écorchage... Ironiquement les appels joyeux de trompe qui sont sonnés ne sont pas des accents de triomphe célébrant la fin monumentale d'un grand rituel, mais un requiem de dérision exécuté pour l'âme de Renart. ${ }^{27}$

Marcelle Thiébaux a mis en lumière une référence implicite au Livre des Seyntz Medecines (1354) de Henry de Lancastre, notamment à travers la figure du renard. Elle résume ainsi la teneur du passage idoine du livre :

Foxes hide in their dens during the day but range abroad by night, seeking their prey. Henry gives the meaning: the evil of the human heart is generally kept concealed from the world's recognition, but at night the vices issue forth privily to do harm. Hunting the fox and routing them out of their hole by various methods constitutes the work, allegorically, of the father confessor...

The retention of the foxes' skin may be allegorized, continues Henry, as the perpetual memory, however painful and shaming, of the sins that are to be kept always before the eyes of the heart. ${ }^{28}$

On voit comment la peau du renard, seul gain de la troisième chasse, équivaut fonctionnellement à la ceinture verte que reçoit Gauvain le même jour, et qui lui servira dans un premier temps à exhiber et à se remémorer sa faute. Bertilak semble en outre remplir à la perfection le rôle du veneur-confesseur dans le poème, même si Gauvain se confesse également et tout d'abord auprès d'un prêtre au château de Hautdesert. La confrontation à la chapelle verte se termine par ce qu'il convient d'appeler une confession, qui suit en première analyse la progression détaillée dans le livre de Henry d'une manière

\footnotetext{
${ }^{27}$ Ibid., p. 121.

${ }^{28}$ Marcelle Thiébaux, op. cit., p. 84.
} 
d'éradiquer les péchés-renards, le chasseur enfonçant dans les trous de terrier une verge composée de trois bâtons liés, appartenant à trois arbres qui ont pour nom : Verray Confession, Dolorous Contricion, Ferme Esperance de Mercy. ${ }^{29}$ Or Gauvain ne traverse clairement que les deux premières de ces trois étapes selon nous, et non les trois comme l'écrit Marcelle Thiébaux. ${ }^{30}$ Bien que le chevalier reconnaisse sa faute, qu'il s'en accuse, s'en repente (v. 2378- 2388), et reçoive le pardon (v. 2391-2394), il estime que l'on devrait excuser celle-ci, car d'autres hommes tels que lui, qu'il cite en puisant dans l'Ancien Testament, ont pareillement péché, trahis par des femmes (v. 2414-2428). Et si Gauvain déclare vouloir conserver la ceinture verte pour combattre son orgueil en des moments d'euphorie de gloire (v. 2433-2438), il n'en reste pas moins que son refus de revenir à Hautdesert et sa manière demeurent ambigus, laissant transparaître la fierté blessée du chevalier, qui se traduit par une obstination à considérer sa faute comme impardonnable, alors qu'il vient d'en être absous. ${ }^{31}$

L'étude des méthodes visant à se défaire du péché envisagées par Henry de Lancastre permettrait certes de mettre au jour un dense réseau de correspondances avec le poème,${ }^{32}$ reposant sur le filage de la métaphore de la

\footnotetext{
${ }^{29}$ Émile J. Arnould (ed.), Le Livre des Seyntz Medecines: the unpublished devotional treatise of Henry of Lancaster, Oxford: Blackwell (Anglo-Norman Texts 2), 1940, p. 113.

30 Marcelle Thiébaux, "Sir Gawain, the fox hunt, and Henry of Lancaster", in Neuphilologische Mitteilungen 1971, pp. 469-479, p. 476.

${ }^{31}$ Nicholas Watson, "The Gawain-poet as a vernacular theologian", in A Companion to the Gawain-Poet, Derek S. Brewer and Jonathan Gibson (eds), Woodbridge, Rochester (New York): D. S. Brewer, 1997, p. 314.

${ }^{32}$ Parmi les plus marquantes que nous relevons à partir du travail de Thiébaux sur le texte de Henry de Lancastre: (1) Les péchés capitaux dérivent de deux principaux péchés-renards, l'orgueil dont Gauvain tend à faire preuve ponctuellement, et la paresse qui conduit à rester au lit et à plonger dans la luxure, ce à quoi on l'invite également ; (2) cinq chiens, représentant les cinq sens, peuvent venir en aide au pécheur dont la vigilance peut lui permettre de débusquer dans la nuit les péchés-renards - les cinq sens, rappelés par le pentacle de Gauvain (v.640), étant tout aussi bien ceux par lesquels se manifeste la vigilance que ceux par lesquels on faillit ; de manière semblable, le veneur confesseur recourt à un chien qui aboie face aux renards à l'intérieur de leur terrier, figurant la conscience qui confesse et livre les péchés ; (3) avec un pécheur impénitent, il peut se révéler nécessaire de recourir à une pique à deux pointes, pour frapper le corps d'une dure pénitence et l'esprit ou l'âme des terreurs de l'enfer (on pense à la hache à double tranchant du chevalier vert, qu'il aiguise avec application).
} 
chasse au renard, animal représentant le mal dans l'imaginaire médiéval, et de façon plus restreinte, ici, le péché. Cela nous éloignerait de notre propos. Nous nous contenterons d'observer que le parallèle entre les deux œuvres permet de comprendre pourquoi Thiébaux estime que la chasse dans Sir Gawain and the Green Knight a une vocation instructive. ${ }^{33}$ En réalité, si la chasse se comprend à un niveau allégorique ou analogique, elle n'est pas directement instructive pour le protagoniste (voire le lecteur), et sa portée lui échappe pleinement. Ce n'est pas sa fonction première. A l'opposé, contrairement à Débax, nous ne voyons pas non plus, de façon minimaliste, le renard et sa roublardise simplement reflétés dans le fait que "Gauvain sait faire d'un défaut un titre de gloire : luimême et toute la cour vont porter la ceinture, cause de la faute, comme un baudrier". ${ }^{34}$ Il y a bien plus.

\section{Tentative de prolongement de l'analogie}

Les similitudes entre l'épisode de la chasse au renard et la troisième tentative de séduction de la châtelaine (ainsi que ses conséquences) sont frappantes. Le renard croit tout d'abord échapper à ses poursuivants (v. 1711), avant de se retrouver face à une nouvelle meute de chiens, de même que Gauvain évite le danger charnel avec l'aide de sa protectrice la Vierge, mais se trouve soumis à la tentation d'un don courtois que lui propose celle qui voudrait être sa dame, et manque finalement de refuser le don du nœud vert. Les chasseurs appellent le renard voleur, "pef" (v. 1725), terme qui convient au chevalier qui s'approprie la ceinture verte sans la restituer à son hôte. Le dernier écart ou la dernière esquive qui conduit le renard à la mort ressemble à l'acceptation par Gauvain du dernier don courtois (la ceinture, après le gant demandé par la châtelaine et l'anneau qu'elle lui offre). Par ce dernier retournement, le renard évite la décapitation, comme le neveu d'Arthur qui s'y

\footnotetext{
${ }^{33}$ Marcelle Thiébaux, The Stag of Love: The Chase in Medieval Literature, Ithaca (NY): Cornell University Press, 1974, p. 71.

${ }^{34}$ Jean-Paul Debax, op. cit., p. 125.
} 
soustrait. De plus, l'écorchage du renard évoque l'atteinte à la peau de son cou, et le requiem de dérision pour l'âme du vil animal mime la chute morale de Gauvain qui, bien qu'il garde sa tête, a tout de même failli. Le rapprochement avec le renard participe ainsi plus globalement de la logique de moquerie visant le chevalier de Camelot.

La comparaison présente aussi un versant plus positif. Gauvain fait régulièrement preuve d'adresse en recourant à des artifices afin de se tirer d'affaire, comme lorsqu'il fait semblant de dormir de sorte à décourager la châtelaine qui s'installe sur lit pour sa première visite (v. 1190). Ce n'est pas la crainte du cerf qui le pousse à se tapir pour éviter l'affrontement, mais la ruse du renard. L'épouse de Bertilak suggère d'ailleurs que c'est l'un des attributs du chevalier, après lui avoir demandé s'il avait une amie et avoir entendu une réponse négative (v. 1786-1787) :'For alle pe lufez vpon lyuve, layne not pe sope / For gile. 35

On peut toutefois légitimement se demander si Gauvain est le seul (et le véritable) renard : le poème indique qu'au début de troisième jour, Bertilak se lève très tôt, et il est alors désigné par le terme seigneur (lord), complémenté par une relative appositive qui signifie à la fois 'qui garde des tours dans son sac' et 'qui prolonge son divertissement' (Pe lord, pat his craftez kepes, v. 1688), deux lectures parfaitement compatibles en contexte. Le plus roublard de l'hôte et de son illustre invité n'est probablement pas celui que l'on croit. De son côté, la châtelaine entre dans la chambre du chevalier parée d'un manteau de fourrure (v. 1736-1737), dont on pourrait s'interroger par rapport à la provenance, d'autant plus que la fourrure de renard est la seule prise de la journée. Il convient au surplus de noter que Bertilak se distingue par sa pilosité de couleur rousse, comme celle du renard (la couleur de sa barbe est comparée précisément à celle

\footnotetext{
${ }^{35}$ Pour tout l'amour qui est sur cette terre ne cachez pas la vérité / Par tromperie.
} 
du castor, animal qui caractérise bien l'énergie de Bertilak (v. 845): Brode, bryzt watz his berdre and al beuer-hwed. ${ }^{36}$

Les deux époux font en tout état de cause preuve de grande fourberie en entreprenant de tendre un piège pernicieux à leur hôte le preux Gauvain, à l'instigation de Morgane.

\section{Chasse et motifs littéraires}

S'intéressant aux scènes de chasse dans le poème en regard de la tradition littéraire, Anne Rooney évite toute interprétation allégorique ou métaphorique des animaux pris par Bertilak. Elle souligne ainsi que lors de la chasse au sanglier, la décision prise de descendre de cheval, de délaisser son épieu pour combattre la bête à pied avec son épée ne fait pas sens d'un point de vue cynégétique, mais se comprend dans le contexte des récits antiques où le héros (comme le jeune Ulysse d'Homère) affronte au corps à corps l'animal, la victoire contribuant de fait à conférer au chasseur une dimension héroïque, voire épique. Elle souligne également que l'épisode de Hautdesert joue sur le motif littéraire familier du chasseur absent dont la femme ou la fille séduit le protagoniste, le lecteur ou l'auditeur s'attendant à cette issue, alors même qu'elle est éludée par Gauvain dans le poème. ${ }^{37}$

Au bas moyen-âge, divers auteurs (comme Henry de Lancastre) ont employé le lieu devenu commun de la chasse comme exercice détournant les hommes du péché, notamment de la paresse, et était considérée comme une excellente préparation au combat, ${ }^{38}$ dont Gauvain se voit privé avant sa rencontre avec le chevalier vert. De plus, comme le note encore Rooney, la poésie allitérative contemporaine (et The Book of the Duchess de Chaucer) a

\footnotetext{
${ }^{36} \mathrm{Sa}$ barbe était large et brillante, elle avait partout la couleur du castor ; [bryzt, traduit par brillant, indique une brillance dans les tons rouge-orangés]

${ }^{37}$ Anne Rooney, op. cit., pp. 157-163.

${ }^{38}$ Sur ce dernier point : Marcelle Thiébaux, op. cit., p. 75.
} 
fréquemment recours aux scènes de chasse. La chasse y représente alors la vie séculaire de cour et le plaisir mondain, que l'on oppose au caractère éphémère de la vie humaine et à l'inévitabilité de la mort, dans le cadre d'un memento mori. ${ }^{39}$ Les plaisirs de la vie de cour, présents également dans les scènes courtoises où Gauvain converse, éloignent les hommes de la vertu en leur permettant de fuir la pensée de leur mort prochaine, qui est toutefois toujours présente à l'esprit du chevalier à Hautdesert. En somme nous soulignerons que le sens des chasses de Bertilak est complexe et riche, contribuant d'une part à suggérer que son invité sombre dans le vice et, d'autre part, que le faste de la vie de cour, à Hautdesert tout aussi bien qu'à Camelot, relève de la vanité. Malgré la fascination qu'elle exerce, le luxe et l'opulence des deux cours peuvent paraitre tapageurs, et en particulier si on les juxtapose au motif des cycles, des saisons et des époques qui passent, si prégnant dans le poème.

\section{CHASSES ET ÉCHOS DES SCÈNES DE CHASSE}

Forts de notre fréquentation des écrits critiques, nous proposons une interprétation personnelle des deux premières scènes de chasse combinées à celles de tentation, après avoir rappelé la composition symétrique générale dans laquelle s'inscrit l'aventure de Hautdesert. Nous montrons ensuite que celui-ci trouve des échos dans deux épisodes qui l'entourent.

\section{Une structure en miroir}

Tandis que Gauvain est le neveu d'Arthur et de Morgane, au service du premier, Bertilak apparaît comme le chevalier servant de Morgane. En réalité, il est légitime de se demander si les deux chevaliers ne partagent pas plus qu'il n'y paraît à première vue. Trois couleurs principales leur sont associées, à des degrés divers : le rouge, l'or, et le vert. Lors de l'irruption du chevalier vert et du départ de Gauvain de Camelot, tous deux portent des vêtements avec des

\footnotetext{
${ }^{39}$ Anne Rooney, op. cit., pp. 161-163.
} 
broderies ou des dessins d'oiseaux (v. 166, 610). ${ }^{40}$ En outre, l'imbrication des scènes de chasse et de séduction laisse entendre que l'un pourrait être l'image inversée de l'autre. Durant l'aventure de Hautdesert, ils échangent leurs gains, voire leurs places, puisque Gauvain reste au château et que le seigneur part, tandis que la châtelaine propose ses faveurs à son illustre invité et non à son époux. Celui-ci se fait chasseur, ses proies sont des bêtes, et non Gauvain, qui lui fait figure de proie par rapport à la châtelaine, qui tente de le piéger comme un animal.

La symétrie des deux personnages se voit amplifiée par la structure en miroir de la narration de l'épisode. Après l'accueil du chevalier d'Arthur et le lancement du jeu de l'échange de gains, chaque journée se déroule suivant la même séquence :

A/ Début bruyant de la chasse de Bertilak, qui a entendu une messe.

B/ Irruption discrète de la châtelaine dans la chambre de Gauvain, et scène de tentation après laquelle il se rend à la chapelle du château.

$\mathrm{C} /$ Fin de la chasse et retour

D/ Échange des gains du jour et festivités

Avec une telle densité d'échos et de jeux de miroir, il paraît pertinent de s'interroger de nouveau quant aux possibles parallèles entre les scènes de chasse et celles de tentation.

\section{Les deux premières journées}

Nous cantonnerons l'analyse aux deux premières journées, puisque nous venons de traiter en détail de la troisième. Au début de la première chasse, des cervidés sont surpris dans un vallon, et seuls les mâles, cerfs et chevreuils peuvent s'enfuir par les hauteurs (un peu comme les chasseurs, qui sont des hommes s'échappant du château), où on les laisse passer suivant la pratique en

\footnotetext{
${ }^{40}$ Je remercie Caroline Lefèvre pour m'avoir soufflé cette remarque.
} 
cette saison. De même, la châtelaine surprend Gauvain en se glissant dans l'alcôve de son lit, qu'il ne peut quitter. Elle lui dit d'ailleurs (v. 1209-1210) :

' 3 e ar a sleper vnslyze pat mon may slyde hider.

Now ar ze tan astyt! ${ }^{41}$

Gauvain est censé être pris, comme les cervidés. Mais contrairement aux cervidés, qui sont d'un naturel craintif, ce qui est reconnu dans les manuels de chasse contemporains, Gauvain dispose d'un courage qui lui permet de se défendre des avances de la dame en gardant à l'esprit, sans chercher à le fuir, le destin qui l'attend (v. 1282-1287):

De freke ferde with defence and feted ful fayre;

pas ho were burde bryztest pe burne in mynde hade,

Pe lasse luf in his lode for lur pat he sozt

Boute hone -

pe dunte pat schulde hym deue,

And nedez hit most be done. ${ }^{42}$

Lors de la deuxième chasse, le sanglier, caractérisé par un orgueil mêlé de témérité (qui le conduit à la mort), est criblé de flèches qu'il méprise. Il devient alors enragé et féroce (v. 1460-1462) :

Bot quen pe dyntez hym dered of her dryze strokez,

Pen, braynwod for bate, on burnez he rasez,

Hurtez hem ful heterly per he forth hyzez; ${ }^{43}$

\footnotetext{
41 "Vous êtes un dormeur imprudent, que l'on puisse ainsi se glisser ici ;

Vous voilà pris sur le champ !"

${ }^{42}$ Le chevalier était sur la défensive et se comportait très bien ;

Quoiqu'elle fût la plus belle des jeunes femmes qu'il ait connu,

Il éprouvait pour elle moins d'amour en raison de la peine qu'il recherchait

Sans délai :

Le coup qui devait l'abattre

Et qui devait nécessairement être frappé.

[Le texte pour les lignes 1283-1284 correspond à notre propre traduction.]

${ }^{43}$ Mais lorsqu'il fut blessé, criblé sous les coups incessants,

Alors fou de douleur dans ce combat il se précipita sur les chevaliers,
} 
Au matin du même jour, la châtelaine fait tôt le siège de Gauvain pour l'aiguillonner (Ful erly ho watz hym ate: v. 1474). Elle présente l'amant courtois, qu'elle voudrait que soit Gauvain, comme un héros capable de subir mille tourments, à l'instar du sanglier qui fait peu cas des flèches (Endured for her drury dulful stoundez: v. 1517). La femme de Bertilak fait ensuite grand cas de la réputation courtoise et surtout amoureuse de Gauvain, en tout cas telle qu'elle est véhiculée dans les romans de chevalerie, et l'invite à faire d'elle son élève. Le chevalier lui répond qu'il n'est pas du tout égal à la tâche (v. 15401545) avec une humilité qui, quoiqu'un peu surfaite du fait de la courtoisie de Gauvain, se situe à l'opposé de l'arrogance et de l'aveuglement que représente le sanglier.

Il semble donc que, contrairement au renard, les deux autres bêtes chassées par Bertilak symbolisent des défauts auxquels Gauvain, en tant que proie potentielle de la châtelaine, échappe largement.

\section{Autres épisodes lus à la lumière des chasses}

Gauvain est plus courageux que les autres chevaliers d'Arthur, qui eux restent en retrait lors de l'irruption du chevalier vert, à la manière de cervidés, restant muets à la question qu'il pose, "C'est qui le chef de cette bande ?" (v. 241-247) :

Perfore to answare watz arze mony apel freke

And al stounded at his steuen and ston-stil seten

In a swoghe silence purz pe sale riche.

As al were slypped vpon slepe so laked hor lotez

In hyze -

I deme hit not al for doute

Les blessant très sauvagement partout où il fonçait. 
Bot sum for cortaysye $-{ }^{44}$

Les chevaliers d'Arthur resteront également interdits lorsque le chevalier vert lancera son défi. Quant au roi, il répond d'abord au chevalier courtoisement, il s'emporte un peu puis lui accorde le don qu'il demande sans en connaître la nature, avant de perdre le sens de la mesure en descendant de son dais pour exprimer sa colère face au chevalier vert. Il représente ainsi assez bien l'orgueil du sanglier. Ses courtisans dénoncent par ailleurs son impétuosité, qui l'a poussé à accorder le don au chevalier vert qui signe la perte de Gauvain, dont ils disent, à l'occasion de son départ (v. 679-684) :

A lowande leder of ledez in londe hym wel semez,

And so had better haf ben pen britned to nozt,

Hadet wyth an aluisch mon, for angardez pryde.

Who knew euer any kyng such counsel to take

As knyztez in cauelaciounz on Crystmasse gomnez? $?^{45}$

Le neveu d'Arthur, qui agit avec une grande habileté, se distingue donc du roi comme de ses pairs : il est le meilleur entre tous.

On pourrait certes reprocher à Gauvain un courage limité puisqu'il craint encore la mort et que son humilité est en partie feinte, faisant partie du jeu courtois. Demeurant humain, il ne peut prétendre à la perfection, et c'est la leçon

\footnotetext{
${ }^{44}$ Aussi plus d'un noble chevalier avait-il peur de répondre, Stupéfaits devant sa voix, pétrifiés sur leur siège, ils étaient là, Et un silence de pâmoison traversait la splendide salle ;

On eût dit qu'ils étaient tombés endormis, tant leur voix était silencieuse,

Soudain,

- Pas tous par peur, je pense,

Quelques-uns par courtoisie -

${ }^{45}$ Il avait l'étoffe d'un illustre meneur d'hommes,

Et cela eût mieux valu pour lui que d'être réduit à néant,

Décapité par un homme qui tenait de l'elfe, tout cela pour un orgueil déplacé.

Vit-on jamais roi prendre pareil conseil :

Des chicaneries de chevaliers lors des jeux de la Nativité ?
} 
principale du roman. Cette impossibilité se traduit par la défaillance temporaire du chevalier lors de la première tentative de décapitation à la chapelle verte : Gauvain tressaille (v. 2267), tout comme un cervidé. De manière semblable, lorsqu'au deuxième essai, le chevalier vert le raille ainsi que la cour de son oncle, Gauvain se montre quelque peu impatient et enjoint son bourreau à accomplir sa besogne, mais il ne s'emporte pas véritablement, à la façon du sanglier, et demeure toujours très pertinent dans ses propos (v. 2284-2301). Le troisième coup, non retenu, ne lui sera pas fatal, mais mettra en lumière sa fourberie toute relative.

Les scènes de chasse se voient donc non seulement réfléchies dans les trois scènes de tentation qui leur font pendant, mais elles renvoient également à l'épisode de l'irruption du chevalier vert à la cour d'Arthur, ainsi qu'à celui de la chapelle verte. Il n'est pas anodin que le motif de la décapitation lie ces épisodes, et que l'on y retrouve la locution schyre grece pour désigner la chair (brillante) tranchée, du cou du chevalier vert (v. 425), des pièces de venaison (v. 1378), et du cou de Gauvain (v. 2313).

Nous conclurons sur un dernier élément qui prolonge et vient clore notre argumentation. La structure en triptyque mise au jour, avec son jeu d'échos et de renvois, se retrouve non seulement dans l'épisode étudié et dans ceux qui l'entoure, où le chevalier vert est présent. Elle est inscrite à l'intérieur même de l'épisode de Hautdesert, puisque lors de la troisième scène de tentation, l'échange de dons que propose la châtelaine constitue une mise en abyme des scènes de chasse. Le premier don qu'elle sollicite est le gant du chevalier, probablement fait en daim, un jeune cervidé faisant partie des animaux abattus au cours de la première chasse. Le deuxième don, qu'elle offre au chevalier, est un anneau. Il se trouve lié à la vanité de la richesse et à l'orgueil, et par là-même au sanglier. Le dernier don est la ceinture verte, gage d'une protection qui se révèle illusoire. C'est un don rusé voire roublard, qui caractérise la personne qui 
l'offre (et celles qui la conduisent à le faire), mais aussi le chevalier qui le reçoit, par l'usage qu'il en attend. Le symbole du renard, tout comme celui de la chasse, est éminemment polysémique. 